\title{
Physics of Fully Depleted CCDs
}

\author{
S. E. Holland; C.J. Bebek, W.F. Kolbe, and J.S. Lee \\ ${ }^{a}$ Lawrence Berkeley National Laboratory \\ 1 Cyclotron Road, Berkeley, CA, 94720, USA \\ E-mail: sehollandelbl.gov
}

\begin{abstract}
In this work we present simple, physics-based models for two effects that have been noted in the fully depleted CCDs that are presently used in the Dark Energy Survey Camera. The first effect is the observation that the point-spread function increases slightly with the signal level. This is explained by considering the effect on charge-carrier diffusion due to the reduction in the magnitude of the channel potential as collected signal charge acts to partially neutralize the fixed charge in the depleted channel. The resulting reduced voltage drop across the carrier drift region decreases the vertical electric field and increases the carrier transit time. The second effect is the observation of low-level, concentric ring patterns seen in uniformly illuminated images. This effect is shown to be most likely due to lateral deflection of charge during the transit of the photogenerated carriers to the potential wells as a result of lateral electric fields. The lateral fields are a result of space charge in the fully depleted substrates arising from resistivity variations inherent to the growth of the high-resistivity silicon used to fabricate the CCDs.
\end{abstract}

KEYWORDS: fully depleted CCD; point spread function; resistivity striations.

${ }^{*}$ Corresponding author. 


\section{Contents}

1. Introduction 囵

2. Effect of signal charge on the point spread function 1]

3. Low-level effects in uniform illumination

母. Summary Q9

\section{Introduction}

Fully depleted charge-coupled devices (CCDs) are presently in use in three major astronomical imaging cameras: the PAN-Starrs PS1 camera [1], the HyperSuprime-Cam camera [2], and the Dark Energy Survey Camera (DECam) [3]. The thicknesses of the CCDs are 75 [4], 200 [5] and $250 \mu \mathrm{m}$ [6], respectively. The primary advantages of the thick, fully depleted devices compared to thinned, conventional scientific CCDs are the enhanced quantum efficiency and reduced fringing at long wavelengths [7].

The purpose of this work is to present first order, physics-based models to explain some of the low-level phenomena that have been observed during telescope operation with these thick, fully depleted CCDs. The effects of interest in this work are the dependence of the CCD point-spread function (PSF) on signal level, and the appearance of fixed-pattern, low-level variations in the $\mathrm{CCD}$ images seen during uniform illumination of the devices. For the former we present a first order theoretical model for the effect, while for the latter we include experimental results to assist in the understanding of the basic mechanisms involved.

The CCDs described in this work are fabricated on high-resistivity silicon substrates that are manufactured using the float-zone refining technique [8]. The resistivity is greater than $4000 \Omega-\mathrm{cm}$ for the n-type substrates considered here, and the CCDs are operated with a substrate-bias voltage that fully depletes the high-resistivity substrate [9]. As discussed in the remainder of the paper, the low-level effects that arise in these thick, fully depleted CCDs depend on the vertical electric fields in the device, and we show that the effects can be reduced by operating the CCDs with increased vertical fields.

\section{Effect of signal charge on the point spread function}

We have previously described simple models for the PSF of fully depleted CCDs [10-14]. The model is based on the lateral diffusion of the photo-generated holes as they are drifted by the vertical electric field in the fully depleted substrate. We briefly review this model and then discuss 


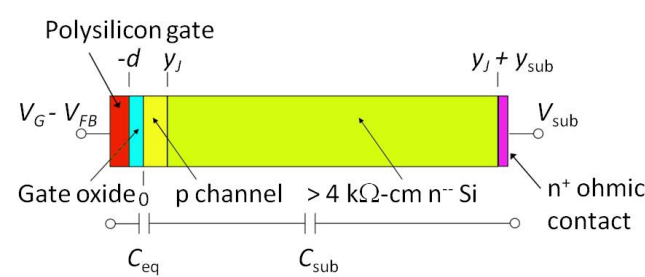

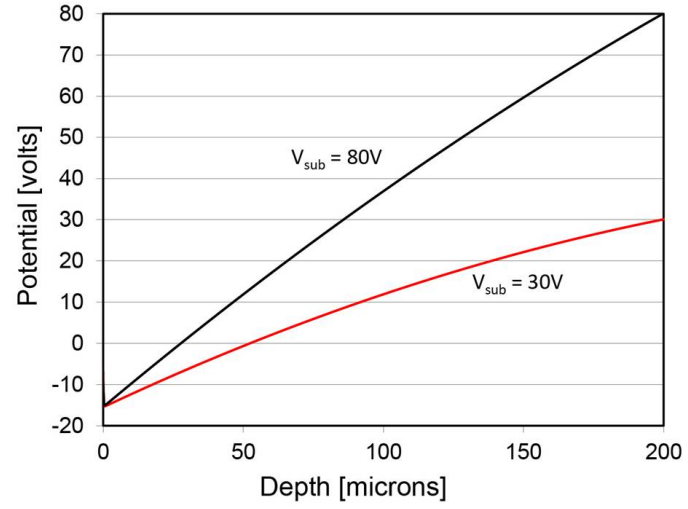

b)

Figure 1. a) Simplified cross-sectional diagram for a fully depleted CCD. b) Simulated electrostatic potential for a $200 \mu \mathrm{m}$ thick CCD at substrate-bias voltages of 30 and $80 \mathrm{~V}$.

enhancements to take into account the effect of signal charge. The PSF in the over-depleted case is given by

$$
P S F_{\text {od }}=\sqrt{2 D t_{\mathrm{tr}}}=\sqrt{2 \frac{k T}{q} \frac{\varepsilon_{\mathrm{Si}}}{\rho_{n}} \ln \frac{E_{\mathrm{y}_{\mathrm{J}}}}{E_{\mathrm{y}_{\mathrm{sub}}}}}
$$

where $D$ and $t_{\text {tr }}$ are the diffusion coefficient and transit time for holes, respectively, $k$ is Boltzmann's constant, $T$ is the temperature in kelvin, $q$ is the electron charge, $\varepsilon_{\mathrm{Si}}$ is the permittivity of silicon, and $\rho_{n}=q N_{D}$ is the volume charge density in the fully depleted substrate where $N_{D}$ is the substrate doping density. Referring to Fig. 四a), $E_{\mathrm{y}_{\mathrm{J}}}$ is the electric field at the p-channel, n-substrate junction located at $y_{J}$, and $E_{\mathrm{y}_{\mathrm{sub}}}$ is the electric field at the back-side contact of the CCD.

The electric field in the fully depleted substrate from a one-dimensional (1-D) solution of the Poisson equation assuming uniform doping density in the channel and substrate is given by [12]

$$
E(y)=\frac{q N_{D}}{\varepsilon_{\mathrm{Si}}}\left(y-y_{J}\right)+E_{J} \quad y_{J}<y<\left(y_{J}+y_{\mathrm{sub}}\right)
$$

where

$$
E_{J} \equiv-\frac{d V}{d y}\left(y_{J}\right)=-\left(\frac{V_{\mathrm{sub}}-V_{J}}{y_{\mathrm{sub}}}+\frac{1}{2} \frac{q N_{D}}{\varepsilon_{\mathrm{Si}}} y_{\mathrm{sub}}\right)
$$

$V_{\text {sub }}$ is the applied substrate-bias voltage, and $V_{J}$ is the electrostatic potential at the p-channel, nsubstrate junction. It was shown in reference [12] that $V_{J}$ is approximately equal to the value of the CCD potential at the potential minimum $V_{m}$, and that $V_{J}$ is approximately independent of $V_{\text {sub }}$ when the substrate doping is much less than the channel doping and the substrate thickness is much greater than the sum of the channel and gate-insulator thickness. A simple capacitor-voltage divider equivalent circuit makes this clear. $C_{\text {sub }}$ in Fig. (1) a) is much less than the equivalent series capacitance $C_{\mathrm{eq}}$ of the channel and gate dielectric, and as a result the channel potential is a weak function of the substrate voltage. 
Figure 1 b) shows the simulated electrostatic potential versus depth for a $200 \mu \mathrm{m}$ thick CCD at substrate-bias voltages of 30 and $80 \mathrm{~V}$. The simulation cross-section was generated from a twodimensional simulator that takes into account the steps used in fabricating the actual devices. The simulation methods are described in more detail below. A key point is that the total voltage drop across the depleted region $V_{\text {sub }}-V_{J} \approx V_{\text {sub }}-V_{m}$ determines the electric field and hence the PSF. In practice when fitting the model to experimental data we have found that the dependence of hole mobility on electric field must be taken into account in order to accurately fit data at high substrate-bias voltages [14].

The model presented above assumes that $V_{J}$ is constant and independent of $V_{\text {sub }}$. While the latter is a good approximation, the former is not valid when charge is stored in the CCD potential wells. Early numerical [15] and analytical [16] solutions to the 1-D Poisson equation that include the signal charge in the CCD potential wells showed that the effect of signal charge is to decrease in magnitude $V_{m}$. We postulate that this effect is the cause of the observed dependence of the PSF on the signal level that has been observed during the operation of the Dark Energy Survey Camera. In order to study this in more detail, we incorporate the effect of the signal charge on $V_{m}$ in the simple PSF model as described below.

The 1-D models described in references $[15,16]$ predict an approximately linear dependence on the signal-charge level for the potential at the $\mathrm{Si}_{-} \mathrm{SiO}_{2}$ interface and at the location of the potential minimum, respectively. An analytic equation for this relationship was given in reference [16] with $V_{m}$ dependent on the channel doping and thickness, the gate-insulator thickness, the substrate doping, the applied gate voltage, and the signal-charge level.

We have studied the effect of signal charge on the channel potential with the aid of 2-D simulation. Figure 2 shows results from the simulations. The process simulator Synopsys TSUPREM-4 was used to generate a realistic cross-section. Photolithography, etching, ion implantation, oxidation, thin-film deposition, and diffusion steps as specified in the actual fabrication sequence were included in the simulation. This cross-section including accurate doping profiles and geometries was then input into the 2-D device simulator Synopsys Medici that solves the Poisson and semiconductor continuity equations in a self-consistent fashion. In addition, the 2-D device simulator can model photo-generation of charge.

The middle panel of Fig. [ 2 shows a portion of the cross-section that was generated and used in the 2-D device simulation. The full cross-section includes 3 pixels at a $10.5 \mu \mathrm{m}$ pitch. Three layers of polysilicon are used in the 3-phase CCDs under study here. The cross-sectional plot shows the spatial distribution of the signal charge represented as a logarithmic contour plot.

The upper portion of Fig. 2 shows the effect of adding signal charge on the electrostatic potential that is shown plotted versus depth along the center line of the collecting phase. The origin of the plot corresponds to the $\mathrm{Si}-\mathrm{SiO}_{2}$ interface. The effect of the signal charge is to electrically neutralize a portion of the depleted channel, i.e. the holes collected in the channel neutralize an equal amount of ionized acceptor atoms resulting in a charge-neutral region where the field is zero. As charge is added to the pixel $V_{m}$ rises. This in turn reduces the voltage drop $V_{\text {sub }}-V_{J} \approx V_{\text {sub }}-V_{m}$ across the drift region which in turn reduces the vertical electric field as shown in the lower portion of Fig. 2. The reduction in vertical electric field causes the hole-transit time to increase resulting in an increase in the PSF. This is the essence of the model that we are presenting here.

To explore the proposed model in more detail, Fig. 3 a) shows the simulated $V_{m}$ versus signal 

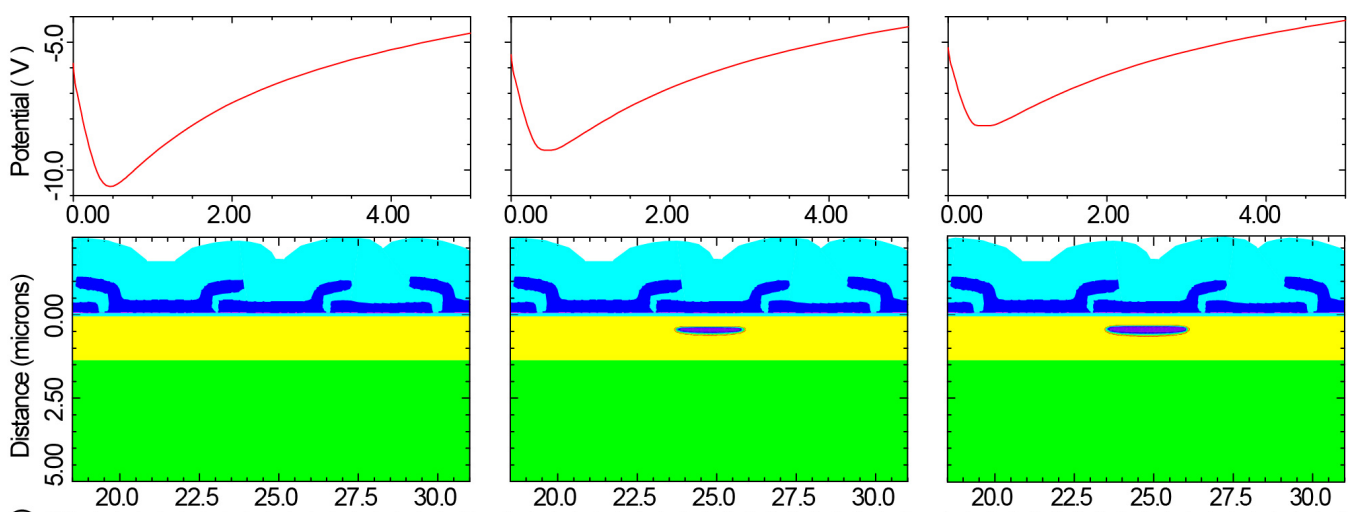

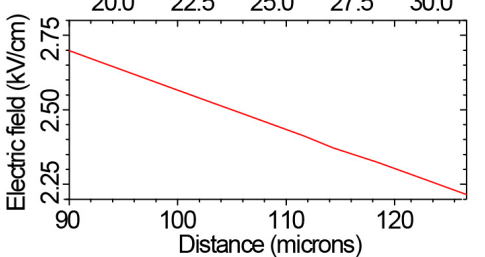

a)

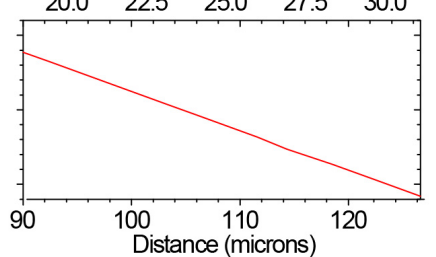

b)

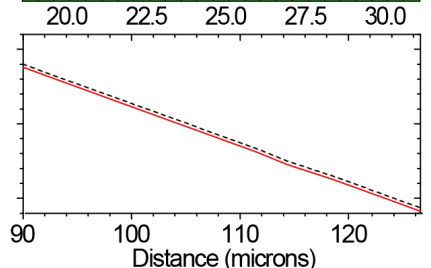

c)

Figure 2. Two-dimensional simulation results demonstrating the change in the vertical electric field (lower plots) and the electrostatic potential near the surface (upper plots) as charge is added to the CCD potential well. The charge levels from left to right are 0,4120 and 8090 holes $/ \mu \mathrm{m}$ width. The center panel shows the simulated device cross-section, and the charge packet of holes is represented as a logarithmic contour plot. The yellow region is the p-type channel, and the green region corresponds to the high-resistivity, n-type substrate. The dark/light blue features are the polysilicon and $\mathrm{SiO}_{2}$ regions, respectively. The potential is plotted versus depth along the center line of the collecting phase. As in the simplified 1-D model, the origin for the potential plots is the $\mathrm{Si}-\mathrm{SiO}_{2}$ interface, and the vertical electric field is plotted over the depth range of 90 to $130 \mu \mathrm{m}$ from the $\mathrm{Si}_{-} \mathrm{SiO}_{2}$ interface. The dashed line in the electric field plot in c) is taken from a), i.e. the case for no signal charge.

charge. Consistent with the earlier 1-D models the simulated $V_{m}$ is an approximately linear function of the signal charge $[15,16]$. Simulations were done at substrate-bias voltages of 50 and $100 \mathrm{~V}$, and as seen in Fig. 3 a) the substrate-bias voltage has a small effect on $V_{m}$ even when signal charge is stored in the pixel.

Figure B b) shows the calculated PSF as a function of signal charge. The plot was generated by calculating the value of $V_{m}$ for a given signal-charge level $q_{s}$ from a linear fit to simulations of the type shown in Fig. 3 a). This value of $V_{m}=f\left(q_{s}\right)$ was then substituted into Eqs. 2.1 through 2.3 to generate the calculated PSF versus $q_{s}$ plot shown in Fig. [ $3 \mathrm{~b}$ ).

The linearity of the predicted PSF versus $q_{s}$ plots shown in Fig. [ B b) improves as the substratebias voltage is increased, and the slope decreases. At large $V_{\text {sub }}$ the space charge due to ionized dopants in the fully depleted substrate can be ignored, and Eq. 2.1] becomes [10,12]

$$
P S F_{\text {od }} \approx \sqrt{2 \frac{k T}{q} \frac{y_{\text {sub }}^{2}}{V_{\text {sub }}-V_{J}}}=\sqrt{2 \frac{k T}{q} \frac{y_{\text {sub }}^{2}}{V_{\text {sub }}}\left(1-\frac{V_{J}}{V_{\text {sub }}}\right)^{-1}}
$$

The last term in the above equation is of the form $1 /(1-x)$ which is approximately equal to $(1+x)$ for $V_{J} \ll V_{\text {sub }}$. The square root of $(1+x)$ is approximately $(1+x / 2)$ for small $x$. With these 


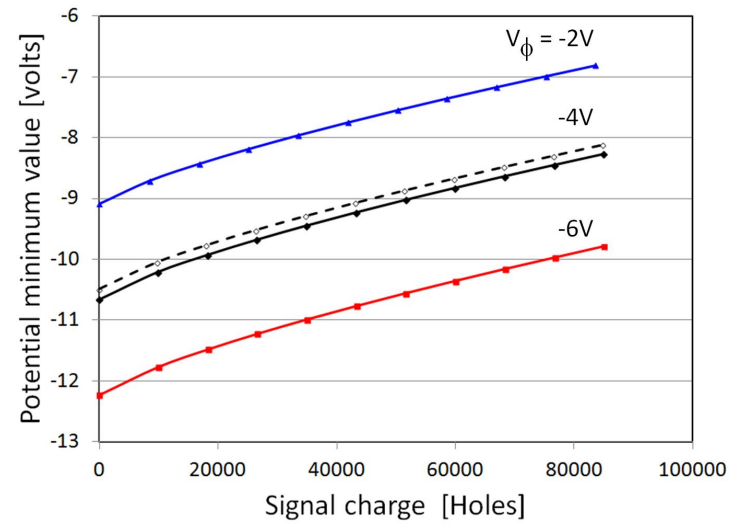

a)

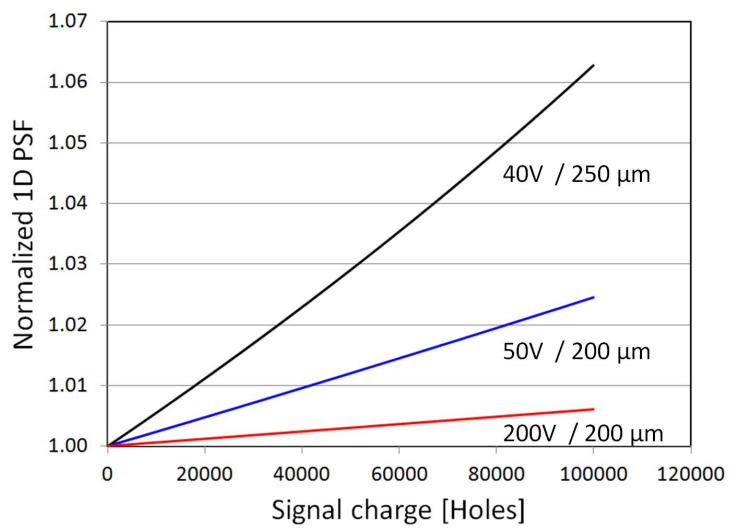

b)

Figure 3. a) Simulated potential-minimum value $V_{m}$ versus signal-charge level for different values of substrate and clock voltages. The solid and dashed lines are for $V_{\text {sub }}=50$ and $100 \mathrm{~V}$, respectively. b) Calculated normalized PSF versus signal-charge level for various combinations of substrate-bias voltage and CCD thickness. The details of the calculation are described in the text. For both plots the simulated charge per unit width was scaled by a width factor of $10.5 \mu \mathrm{m}$.

approximations Eq. 2.4 becomes

$$
P S F_{\text {od }} \approx \sqrt{2 \frac{k T}{q} \frac{y_{\mathrm{sub}}^{2}}{V_{\mathrm{sub}}}}\left(1+\frac{1}{2} \frac{V_{J}}{V_{\mathrm{sub}}}\right) \approx \sqrt{2 \frac{k T}{q} \frac{y_{\mathrm{sub}}^{2}}{V_{\mathrm{sub}}}}\left(1+\frac{1}{2} \frac{V_{m}}{V_{\mathrm{sub}}}\right) \quad V_{J} \ll V_{\mathrm{sub}}
$$

which yields a linear dependence on $V_{m}$. Since $V_{m}$ is approximately a linear function of the signal charge $q_{s}$, it follows that the PSF is approximately a linear function of $q_{s}$ as well when the simplifications described above are valid. The predicted slope of the PSF versus $q_{s}$ plot is then

$$
\frac{d\left(P S F_{\text {od }}\right)}{d q_{s}} \approx \beta \frac{y_{\mathrm{sub}}}{V_{\mathrm{sub}}} \sqrt{\frac{1}{2} \frac{k T}{q V_{\mathrm{sub}}}}
$$

where $\beta$ is the slope of the $V_{m}$ versus $q_{s}$ relationship that depends on the channel doping and other parameters as described earlier.

The model presented above is clearly a simplified view of the situation, and regrettably we do not have experimental data at the present time. Nonetheless, we present the model in order to hopefully elucidate the basic physics of the problem. The model does explain qualitatively the effect of increasing signal charge leading to increased PSF, and does predict that these effects will be a function of the CCD thickness and applied substrate voltage. A more accurate model would take into account the cumulative degradation of the PSF as signal charge is added.

\section{Low-level effects in uniform illumination}

The CCDs described in this work have been shown to exhibit low-level variations in the signal level when illuminated uniformly with light. Figure $\$$ a) shows an image resulting from the medianfiltering of 100 exposures taken with a $4114 \times 2040,250 \mu \mathrm{m}$ thick, $15 \mu \mathrm{m}$ pixel, fully depleted CCD that was uniformly illuminated with $500 \mathrm{~nm}$ light. A fixed-pattern feature consisting of 


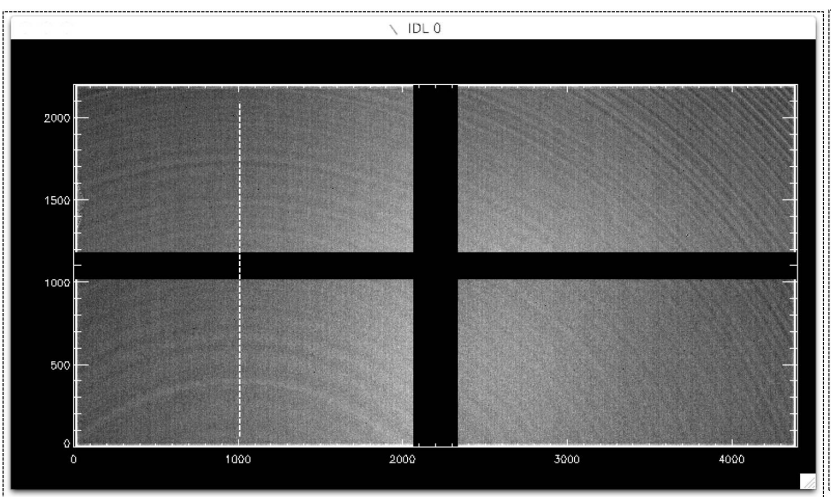

a)

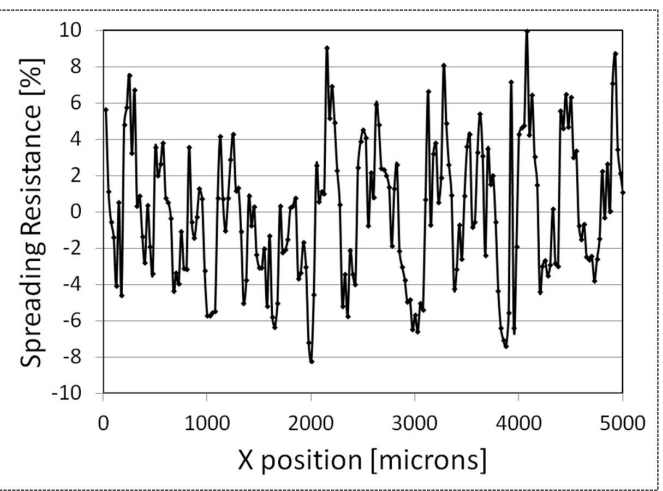

b)

Figure 4. a) Processed image taken with a $4114 \times 2040,250 \mu \mathrm{m}$ thick, $15 \mu \mathrm{m}$ pixel, fully depleted CCD. The substrate-bias voltage was $50 \mathrm{~V}$, and the operating temperature was $-140{ }^{\circ} \mathrm{C}$. The CCD was uniformly illuminated with $500 \mathrm{~nm}$ light, and 100 images were median filtered to generate the picture shown. The mean light level was about 30,000 photons/pixel. The vertical dotted line at column 1000 denotes the radial direction used in the analysis described in the text. b) The percent variation in spreading resistance measured on a $300 \Omega$-cm, n-type, float-zone refined sample. The step size for the 2-point probe measurement was $25 \mu \mathrm{m}$.

concentric rings is visible in the image that has been displayed with enhanced contrast. As shown later, the variations in the image are on the order of a percent.

Similar fixed patterns have been reported for n-channel, fully depleted CCDs [17] and other silicon-based imagers including partially depleted, back-illuminated silicon vidicons $[18,19]$ and front-illuminated commercial CCDs [20,21], Charge Priming Devices [22], and CMOS image sensors [23]. Concentric patterns that represent the error in position location for charged particles in silicon drift detectors have also been reported [24-26]. In all cases the underlying mechanism for the fixed pattern is variations in the resistivity in the bulk silicon substrates that are inherent to the crystal growth processes. Dopant impurities are incorporated into the silicon during crystal growth from the molten phase according to the impurity segregation (distribution) coefficient defined as the ratio of impurity solubility in the solid and liquid phases of the silicon [8,27]. Growth-rate fluctuations due to thermal asymmetries in the melt and temporal variations in temperature that are affected by the crystal rotation and pull rates result in the resistivity striations [28,29].

The concentricity of the rings comes about from the slicing into wafers of the bulk ingots transverse to the growth direction where the growth surface, i.e the crystal-melt interface is approximately spherical in shape $[28,29]$. As a result it is typically observed that the spatial frequency of the striations increases towards the outer portion of the wafer $[21,22,30]$. This effect can be seen in the upper right corner of the image shown in Fig. 7 a). Also, the resistivity varies in the vertical dimension as a consequence of the shape of the growth surface. The resistivity striations are typically characterized by spreading-resistance measurements [29-32]. Fig. 田) shows measurements taken on a $300 \Omega$-cm, n-type, float-zone refined sample that is believed to be qualitatively similar to the silicon used in this work [33].

The concentric fixed patterns in the imagers mentioned previously have been attributed to lateral electric fields that displace the charge carriers [19,20], recombination centers and crystal 
defects that affect the amount of charge collected [20,23], and resistivity-induced variations in the potential barrier to the substrate for the imagers that utilize a vertical-overflow drain [21,22]. Of those effects, it is expected that the lateral electric fields will dominate in fully depleted CCDs as is the case for the silicon drift detectors [24-26]. The float-zone refined silicon used in this work has very few crystalline defects and carrier traps, and the fully depleted operation reduces the possibility of recombination.

In references $[19,20]$ the lateral electric field in the undepleted silicon was modeled as a builtin field that arises from the detailed balance of diffusion and drift currents in thermal equilibrium. The impurity gradient generates a diffusion current that is balanced by the field that arises when charge carriers diffuse away from their origin leaving behind ionized dopant atoms [34]. The field in that case is given by $E_{\mathrm{x} \text {, undepleted }} \approx(k T / q)(1 / x) \ln \left(N_{\mathrm{D} 1} / N_{\mathrm{D} 2}\right)$ where $x$ is the lateral spatial coordinate over which the doping density varies from $N_{\mathrm{D} 1}$ to $N_{\mathrm{D} 2}$.

For the case of a fully depleted device, the effect of the resistivity variation is to add a lateral variation to the volume charge density $\rho_{n}=q N_{D}$ where $N_{D}$ is now a function of $x$. Due to the complexity of solving the 2-D Poisson equation analytically, one must resort to numerical methods in order to accurately model the lateral electric field $E_{x}$ due to the resistivity striations $[35,36]$. We qualitatively estimate the lateral-charge displacement in a fully depleted CCD by first calculating the transverse distance traveled by the holes due to an assumed constant lateral field $E_{x}$ during the transit time $t_{t r}$ of the holes from their generation point to the collection well, i.e.

$$
v_{x}=\frac{d x}{d t}=\mu_{p} E_{x}
$$

where $v_{x}$ is the hole velocity in the lateral direction $x$. After separation of variables and integrating, Eq. 3.1 yields the lateral displacement $x_{L}$ during the time $t_{t r}$

$$
x_{L}=\mu_{p} E_{x} t_{t r}
$$

The transit time neglecting the space charge in the substrate is

$$
t_{t r}=\frac{y_{\text {sub }}^{2}}{\mu_{p}\left(V_{\text {sub }}-V_{J}\right)}
$$

The fractional, lateral displacement of the holes is then solved for by substituting Eq. 3.3 into Eq. 3.2 yielding

$$
\frac{x_{L}}{y_{\text {sub }}}=\frac{E_{x} y_{\text {sub }}}{V_{\text {sub }}-V_{J}} \approx \frac{E_{x}}{E_{y}}
$$

A similar argument was presented in reference [20] for the case of the built-in field that applies in undepleted regions.

This simple model predicts that the amplitude of the deviations of the light level from the mean value are independent of temperature and can be reduced by increasing the substrate-bias voltage. The residual-light level should also be wavelength dependent, with shorter wavelength light showing the largest effect since the photo-generated holes travel the longest distance in this case and will be subject to the lateral fields for a longer period of time. In the following we explore the validity of Eq. 3.4 by comparing the predictions to experimental results. 


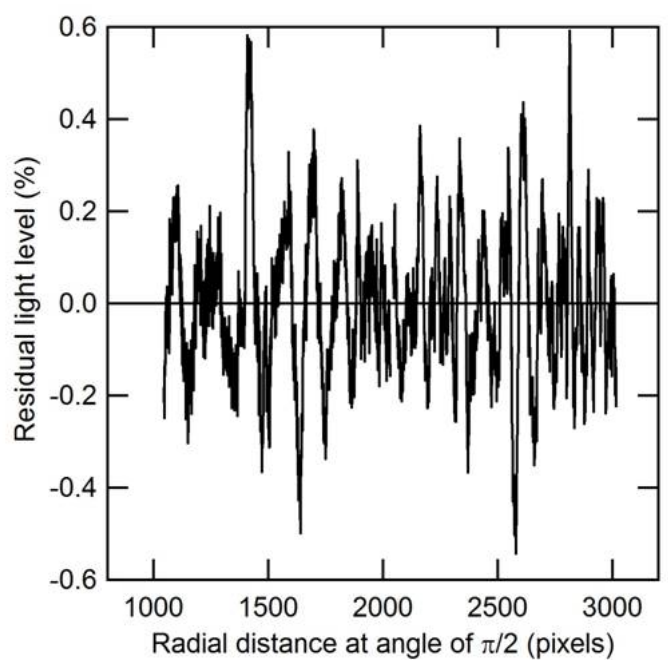

a)

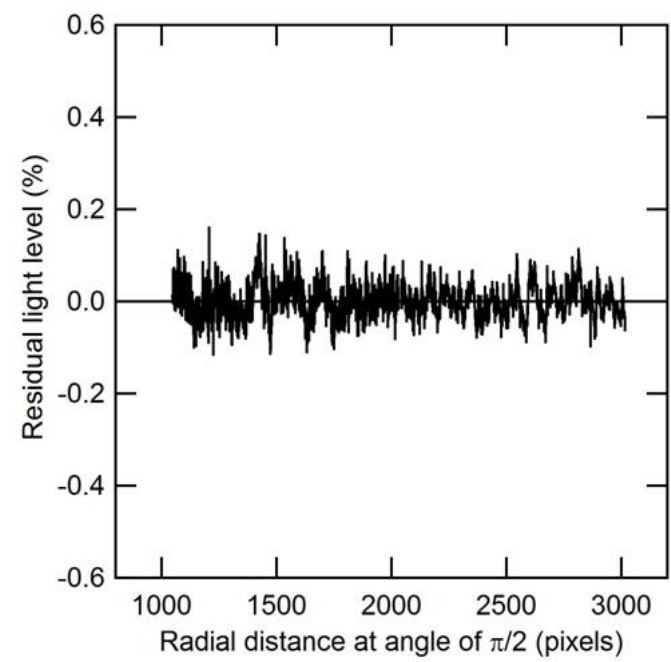

b)

Figure 5. The residual-light level in percent measured at two substrate-bias voltages on a $4114 \times 2040$, $250 \mu \mathrm{m}$ thick, $15 \mu \mathrm{m}$ pixel CCD. The operating temperature was $-140{ }^{\circ} \mathrm{C}$. a) $V_{\text {sub }}=40 \mathrm{~V}$. b) $V_{\text {sub }}=150 \mathrm{~V}$.

In order to improve the statistics of the measurements, median-filtered images generated from 100 individual exposures were used for the analysis. The images were then converted to polar coordinates to transform the circular patterns into linear ones that were then averaged over 500 pixels for each data point shown in the following. The conversion to polar coordinates changes the row, column pixel coordinates to $(r, \theta)$, and the averaging is then done over a range of angles centered at $\theta=\pi / 2$ over the radial distance $r$ for the data presented below. The angle $\pi / 2$ corresponds to the dotted line at about column 1000 in Fig. 6 a).

Figure 5 shows the residual-light level in percent for substrate-bias voltages of 40 and $150 \mathrm{~V}$. The operating temperature was $-140{ }^{\circ} \mathrm{C}$. We have also performed measurements at $-100{ }^{\circ} \mathrm{C}$ and do not see a significant temperature dependence. The residual-light level was calculated by first subtracting a smooth curve from the raw data. This was necessary in order to account for small non-uniformities in the light intensity that was used to illuminate the CCD. A quadratic, leastsquares fit was used to generate the smooth curve. The residual value in analog-to-digital units was then divided by the average light level calculated from the quadratic-fit curve to generate the data shown in Fig. 5. As predicted by Eq. 3.4 the amplitude of the residual-light level is reduced as $V_{\text {sub }}$ is increased due to the reduction in transit time at higher $E_{y}$ that in turn reduces the time that the holes are exposed to the lateral fields due to the resistivity variations. It must be noted that the CCDs described in this work were specifically designed to operate reliably at high substrate-bias voltages $[37,38]$.

Eq. 3.4 predicts that the fractional charge displacement should decrease as $1 /\left(V_{\text {sub }}-V_{J}\right)$. Figure 6 a) shows the measured residual-light level in percent showing three regions where the peakto-peak amplitudes were extracted at each substrate-bias voltage. A binomial smoothing algorithm was used to find the minimum and maximum values for each region. Figure $6 \mathrm{~b}$ ) shows a plot of the extracted amplitude of the residual-light level in percent as a function of inverse substrate-bias 


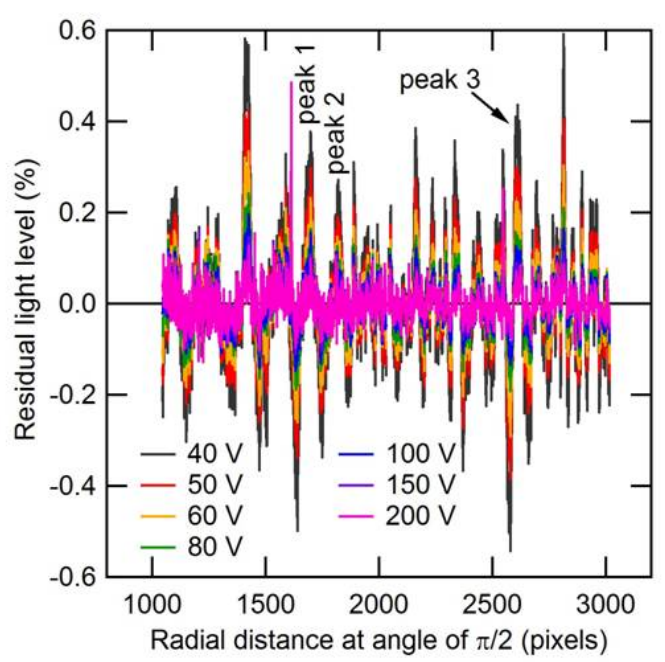

a)

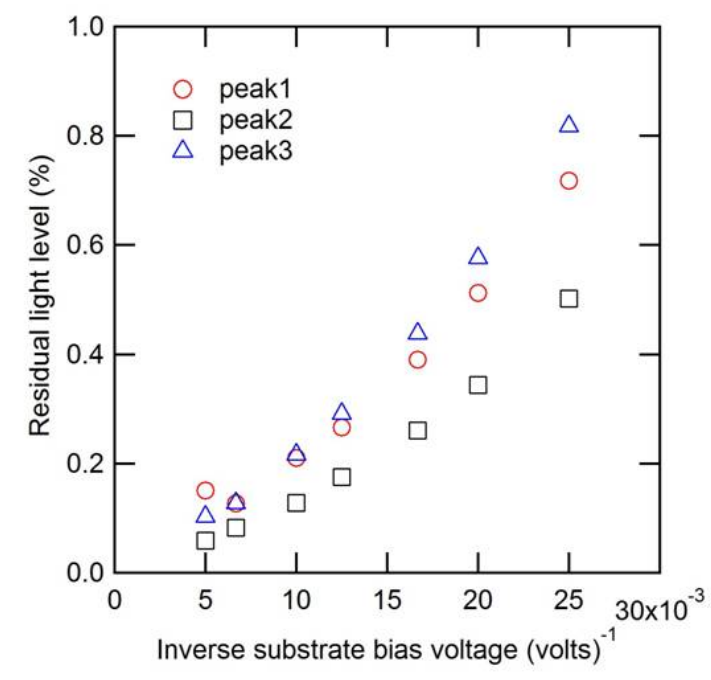

b)

Figure 6. a) The residual-light level in percent measured at different substrate-bias voltage levels on a $4114 \times 2040,250 \mu \mathrm{m}$ thick, $15 \mu \mathrm{m}$ pixel CCD. b) The residual-light level peak-to-peak amplitude versus inverse substrate-bias voltage calculated for peaks 1 to 3 in a).

voltage over the $V_{\text {sub }}$ range of 40 to $200 \mathrm{~V}$. The data trend in qualitative agreement with the simple model presented above. The current model neglects the spatially varying transverse diffusion due to the resistivity striations and the effect of the vertical electric field on the hole mobility. Also, the assumption of a constant $E_{x}$ is an oversimplification [36]. These effects will be incorporated in future studies. Nonetheless the simple model does point towards two means to reduce the effect: operation at high vertical electric fields, and the use of the highest resistivity silicon that is practical in order to reduce the magnitude of the lateral fields.

\section{Summary}

We have presented a simple, physics-based model to explain the dependence of the point-spread function on light level in fully depleted CCDs. Given that this work is part of the proceedings of a workshop, it is not intended that this model be considered a rigorous solution to the problem but rather a starting point for a more accurate model. We have also studied the low-level variations in signal level seen during uniform illumination of fully depleted CCDs, and have shown through a review of the literature that essentially all types of silicon imaging devices can exhibit similar effects. The physics of the observed effects for the various types of imagers differ, but in all cases the origin of the low-light variations is the resistivity striations that are inherent to the growth of the silicon crystals. We have presented a simple model based on the lateral displacement of charge due to lateral volume charge density variations arising from the resistivity striations, and have presented experimental evidence in support of the model. The operation of fully depleted CCDs at high vertical electric fields offers a means to minimize both effects considered in this paper. 


\section{Acknowledgments}

The CCDs used in this work were packaged by John Emes. We would like to thank the anonymous reviewer who made us aware of the prior work on silicon drift detectors. This work was supported by the Director, Office of Science, of the U.S. Department of Energy under Contract No. DEAC02-05CH11231. The U.S. Government retains, and the publisher, by accepting the article for publication, acknowledges, that the U.S. Government retains a non-exclusive, paid-up, irrevocable, world-wide license to publish or reproduce the published form of this manuscript, or allow others to do so, for U.S. Government purposes.

\section{References}

[1] N. Kaiser et al., "The Pan-STARRS wide-field optical/NIR imaging survey," Proc. of SPIE, 7733, $77330 \mathrm{E}-1,2010$.

[2] Y. Komiyama et al., "Hyper Suprime-Cam: Camera Design," Proc. of SPIE, 7735, 77353F-1, 2010.

[3] B. Flaugher et al., "The Dark Energy Camera (DECam)," Proc. of SPIE, 7014, 70140E-1, 2008.

[4] J.L. Tonry, B.E. Burke, S. Isani, P.M. Onaka, and M.J. Cooper, "Results from the Pan-STARRS Orthogonal Transfer Array (OTA)," Proc. of SPIE, 7021, 702105, 2008.

[5] Y. Kamata, S. Miyazaki, H. Nakaya, H. Suzuki, Y. Miyazaki, and M. Muramatsu, "Characterization and Performance of Hyper Suprime-Cam CCD," Proc. of SPIE, 7742, 774229-1, 2010.

[6] J. Estrada et al., "Focal Plane Detectors for Dark Energy Camera (DECam)," Proc. of SPIE, 7735, 77351R-1, 2010.

[7] D.E. Groom, S.E. Holland, M.E. Levi, N.P. Palaio, S. Perlmutter, R.J. Stover, and M. Wei, "Quantum efficiency of a back-illuminated CCD imager: an optical approach,” Proc. of SPIE, 3649, 80, 1999.

[8] W. von Ammon and H. Herzer, "The production and availability of high resistivity silicon for detector application," Nucl. Instrum. and Meth. A, bf 226, 94, 1984.

[9] S.E. Holland, G. Goldhaber, D.E. Groom, W.W. Moses, C.R. Pennypacker, S. Perlmutter, N.W. Wang, R.J. Stover, and M. Wei, "A $200 \times 200$ CCD image sensor fabricated on high-resistivity silicon," in IEDM Technical Digest, 911, 1996.

[10] S.E. Holland et al., "Development of back-illuminated, fully-depleted CCD image sensors for use in astronomy and astrophysics," presented at the 1997 IEEE Workshop on Charge-Coupled Devices and Advanced Image Sensors, Bruges, Belgium, 1997.

[11] D.E. Groom et al., "Point-spread function in depleted and partially depleted CCDs," in Proc. 4th ESO Workshop on Optical Detectors for Astronomy, Garching, Germany, 13-16 September 1999.

[12] S.E. Holland, D.E. Groom, N.P. Palaio, R.J. Stover, and M. Wei, "Fully depleted, back-illuminated charge-coupled devices fabricated on high-resistivity silicon," IEEE Trans. Elec. Dev., 50, 225, 2003.

[13] A. Karcher, C.J. Bebek, W.F. Kolbe, D. Maurath, V. Prasad, M. Uslenghi, and M. Wagner, "Measurement of lateral charge diffusion in thick, fully depleted, back-illuminated CCDs," IEEE Trans. Nucl. Sci., 51, 2231, 2004.

[14] J.A. Fairfield, D.E. Groom, S.J. Bailey, C.J. Bebek, S.E. Holland, A.Karcher, W.F. Kolbe, W. Lorenzon, and N.A. Roe, "Improved spatial resolution in thick, fully depleted CCDs with enhanced red sensitivity," IEEE Trans. Nucl. Sci., 53, 3877, 2006. 
[15] W.H. Kent, "Charge distribution in buried-channel charge-coupled devices," Bell Sys. Tech. Jour, 52, 1009, 1973.

[16] H. El-Sissi and R.S.C. Cobbold, "One-dimensional study of buried-channel charge-coupled devices," IEEE Trans. Elec. Dev., 21, 437, 1974.

[17] I.V. Kotov, A.I. Kotov, J. Frank, P. Kubanek, M. Prouza, P. O'Connor, V. Radeka, and P. Takacs, "Study of pixel area variations in fully depleted thick CCD," Proc. of SPIE, 7742, 774206-1, 2010.

[18] M.H. Crowell and E.F. Labuda, "The silicon diode array camera tube," Bell Sys. Tech. Jour., 48, 1481, May-June 1969.

[19] S. Yoshikawa and J.-i. Chikawa, "Electrical effect of growth striations in the silicon vidicon-type camera tubes," Appl. Phys. Lett., 23, 636, 1973.

[20] L. Jastrzebski, P.A. Levine, A.D. Cope, W.N. Henry, and D.F. Battson, "Material limitations which cause striations in CCD imagers," IEEE Trans. Elec. Dev., 27, 1694, 1980.

[21] Y. Hiroshima et al., "Elimination of fixed pattern noise in Super-8 format CCD image sensor by the use of epitaxial wafers," in IEDM Technical Digest, 32, 1984.

[22] K. Senda, Y. Hiroshima, S. Matsumoto, T. Kuriyama, M. Susa, S. Terakawa, and T. Kunii, "Fixed pattern noise in the solid-state imagers due to the striations in Czochralski silicon crystals," J. Appl. Phys., 57, 1369, 1985.

[23] A. Rantzer and C. Svensson, "Bulk wafer defects observable in vision chips," in $32^{\text {th }}$ European Solid-State Device Research Conference, 24-26 September 2002.

[24] D. Nouasi et al., "Correction of dopant concentration fluctuation effects in silicon drift detectors," Nucl. Instrum. and Meth. A, 461, 222, 2001.

[25] D. Nouasi et al., "The ALICE silicon drift detector system," Nucl. Instrum. and Meth. A, 501, 119, 2003.

[26] E. Crescio et al., "Results from beam tests of large area silicon drift detectors," Nucl. Instrum. and Meth. A, 539, 250, 2005.

[27] J.A. Burton, R.C. Prim, and W.P. Slichter, "The distribution of solute in crystals grown from the melt. Part I. Theoretical," J. Chem. Phys., 21, 1987, 1953.

[28] K. Morizane, A. Witt, and H.C. Gatos, "Impurity distribution in single crystals III. Impurity heterogeneities in single crystals rotated during pulling from the melt," J. Electrochem. Soc., 114, 738, 1967.

[29] T. Abe, Y. Abe, and J.-i. Chikawa, "The structure and origin of growth striations in dislocation-free Si crystals," in Semiconductor Silicon 1973, eds. H.R. Huff and R.R. Burgess (Electrochemical Society, Princeton), 95, 1973.

[30] J.J. Kopanski, J.R. Lowney, D.S. Miles, D.B. Novotny, and G.P. Carver, "High spatial resolution mapping of resistivity variations in semiconductors," Solid-State Elec., 35, 423, 1992.

[31] J. Burtscher, H.W. Dorendorf, and J. Krausse, "Electrical measurement of resistivity fluctuations associated with striations in silicon crystals," IEEE Trans. Elec. Dev., 20, 702, 1973.

[32] A. Mühlbauer, F. Sedlak, and P. Voss, "Investigation of breakdown and resistivity striations in high-voltage silicon diodes," J. Electrochem. Soc., 122, 1113, 1975.

[33] Data courtesy of Leif Jensen, Topsil Semiconductor Materials. 
[34] R. S. Muller and T.I. Kamins, Device electronics for integrated circuits, John Wiley and Sons, Inc., 2nd editon, Section 4.1, New York 1986.

[35] J. Cornu and R. Sittig, "The influence of doping inhomogeneities on the reverse characteristics of semiconductor power devices," IEEE Trans. Elec. Dev., 22, 108, 1975.

[36] I.V. Kotov, T.J. Humanic, D. Nouais, J. Randel, and A. Rashevsky, "Electric fields in nonhomogeneously doped silicon. Summary of simulations," Nucl. Instrum. and Meth. A, 568, 41, 2006.

[37] S. Holland et al., "High-voltage compatible, fully depleted CCDs," Proc. of SPIE, 6276, 62760B-1, 2006.

[38] S.E. Holland, W.F. Kolbe, and C.J. Bebek, "Device design for a 12.3-Megapixel, fully depleted, back-illuminated, high-voltage compatible charge-coupled device," IEEE Trans. Elec. Dev., 56, 2612, 2009. 